\title{
Transcriptome analysis revealed the effect of a combination of red and blue LEDs on photosynthesis, chlorophyll and carotenoid biosynthesis in Brassica campestris L.
}

\author{
T. ZHANG, Y.Y. SHI, F.Z. PIAO, N.S. DU, and Z.Q. SUN ${ }^{+}$ \\ College of Horticulture, Henan Agricultural University, No. 95 Wenhua Road, Zhengzhou, 450002, China
}

\begin{abstract}
Here, we analyzed the transcriptomes of Brassica campestris L. ssp. chinensis Makino under the treatment of a combination of red (R) and blue (B) light. In total, 5,643 differentially expressed genes (DEGs) were found under the R and B combination compared with the white light. An analysis of the DEGs enriched in photosynthesis and antenna proteins showed that there were many more upregulated subunits in PSII than those in PSI. The DEGs encoding the cytochrome $b_{6} f$ complex, photosynthetic electron transport, ATP synthases, and key genes involved in chlorophyll (Chl) and carotenoids (Car) biosynthesis were also upregulated. In addition, real-time PCR indicated that 13 of 15 genes showed the similar expression patterns with the RNA-seq data. The R and B combination might promote photosynthesis by specifically regulating Chl biosynthesis, thus accelerating the growth and development of plants and providing a valuable reference for vegetable production in factories and variety breeding.
\end{abstract}

Additional key words: light-emitting diodes, non-heading Chinese cabbage, PSII, PSI, RNA sequencing.

\section{Introduction}

Light is an important environmental factor and plays key roles in plant growth and morphology (McDonald 2003). Different light spectrum can produce diverse morphological and physiological responses in plants (Wang et al. 2016). The effects of light intensity on plant physiological response were conducted (Acock et al. 1978, Corré 1983). Light-emitting diodes (LEDs) have a long life, high brightness, are environment friendly, and their spectrum can be manipulated (Pimputkar et al. 2009). Furthermore, LEDs can efficiently promote plant growth and development. Plant morphology and physiology are strongly influenced by light quality, such as color and wavelength (Johkan et al. 2010). Red light (R) and blue light (B) are the two most attractive spectrum wavelengths for plants (Liu et al. 2011, Chen 2014). $\mathrm{R}$ influences photosynthetic apparatus, Chl content, stem elongation, and root to shoot ratio (Appelgren 1991, Aksenova et al. 1994, Sæbø et al. 1995). B causes physiological responses via phototropins, including phototropism, chloroplast movement, hypocotyl elongation, leaf expansion, stomatal opening, leaf anatomy, enzyme synthesis, and gene expression (Christie 2007, Inoue et al. 2008, Wang et al. 2009). Compared to white light or a combination of $\mathrm{R}$ and $\mathrm{B}$, exposure to $\mathrm{R}$ or $\mathrm{B}$ alone resulted in an abnormal leaf morphology, a reduced photosynthetic rate $\left(P_{\mathrm{N}}\right)$, and reduced photosynthetic capacity $\left(P_{\max }\right)$, accompanied by changes in Chl content per area, leaf mass per unit leaf area (LMA), nitrogen content per area, and stomatal conductance (Kim et al. 2004, Wang et al. 2009, Hogewoning et al. 2010, Wang et al. 2015, 2016). Plant growth and development require a rational combination ratio of $\mathrm{R}$ and $\mathrm{B}$. Plants under this combination showed the highest $P_{\mathrm{N}}, P_{\max }, \mathrm{LMA}$, and Chl content among all the $\mathrm{R}$ and $\mathrm{B}$ combinations, although the optimal $\mathrm{R}$ and $\mathrm{B}$ ratio varied among different plants (Nhut et al. 2003, Hogewoning et al. 2010, Li et al. 2013, Hernández and Kubota 2016, Wang et al. 2016).

Photosynthesis is the primary metabolic process that converts light energy into chemical energy and drives plant growth and biomass production. Photosynthesis in plants is accomplished by a series of reactions that mainly occur in the chloroplast. Early biochemical studies have shown that chloroplast thylakoid membranes oxidize $\mathrm{H}_{2} \mathrm{O}$, reduce NADP, and synthesize ATP. These reactions are catalyzed by two photosystems, including PSI and PSII. ATP synthase (F-ATPase), which produces ATP at the expense of the proton motive force that is formed by light-driven electron-transfer reactions. The cytochrome

\footnotetext{
$\overline{\text { Received }} 5$ January 2018, accepted 1 August 2018.

${ }^{+}$Corresponding author; e-mail: sunzhiqiang1956@sina.com

Abbreviations: B - blue; Car - carotenoids; Chl - chlorophyll; $C_{\mathrm{i}}$ - intercellular $\mathrm{CO}_{2}$ concentration; DEGs - differentially expressed genes; $E$ - transpiration rate; $g_{\mathrm{s}}$ - stomatal conductance; LEDs - light-emitting diodes; LMA - leaf mass per unit leaf area; $P_{\max }-$ photosynthetic capacity; $P_{\mathrm{N}}$ - photosynthetic rate; R - red; RNA -Seq - RNA sequencing; VDE - violaxanthin de-exposidase; W white; ZEP - zeaxanthinepoxidase.

Acknowledgement: This work was financially supported by the China Earmarked Fund for Modern Agro-industry Technology Research System (CARS-25-C-06).
} 
$b_{6} f$ complex mediates electron transport between PSII and PSI and converts redox energy into the proton gradient that is used for ATP formation (Green and Durnford 1996, Nelson and Yocum 2006). PSII and PSI have their own distinct reaction centers, $\mathrm{P}_{680}$ and $\mathrm{P}_{700}$, respectively, after the wavelengths (in $\mathrm{nm}$ ) of their red-peak absorption maximum (Caffarri et al. 2014). The process of photosynthesis has been studied thoroughly and has greatly benefited from genetic approaches and transcriptomic and proteomic studies (Eberhard et al. 2008, Kaiser et al. 2015). However, the mechanism underlying the regulation of plant photosynthesis by the combination of $\mathrm{R}$ and $\mathrm{B}$ is not fully understood.

Light plays important roles in plant growth and development, particularly in vegetable factories, where $\mathrm{R}$ and $\mathrm{B}$ light are widely applied by light-emitting diodes (LEDs). However, the mechanism by which an R and B combination regulates growth and development is not fully understood. Non-heading Chinese cabbage (Brassica campestris L. cv. Shanghaiqing) originated from China and is an important leafy vegetable grown in Eastern Asia; Non-heading Chinese cabbage is a cultivated variety of this cabbage that is grown in Eastern China. A previous study investigating the effects of $\mathrm{R}$ and $\mathrm{B}$ on the budding and flowering of non-heading Chinese cabbage showed that $\mathrm{B}$ was conducive to vegetative growth, while $\mathrm{R}$ and the combination of $\mathrm{R}$ and $\mathrm{B}$ are beneficial for reproductive growth ( $\mathrm{Li}$ and $\mathrm{Lu} 2016$ ). In this paper, the transcriptome of cabbage was analyzed by RNA sequencing (RNA-Seq) to elucidate the effects of LED R and B combination on photosynthesis in non-heading Chinese cabbage and possibly unravel the mechanism by which the $R$ and $\mathrm{B}$ combination regulates photosynthesis, growth, and development in plants.

\section{Materials and methods}

Plant material: Seeds of non-heading Chinese cabbage (Brassica campestris L. cv. shanghaiqing) were sown in rock-wool blocks $(25 \times 25 \times 40 \mathrm{~mm})$ and were cultivated in a solar greenhouse for one month. The environmental conditions were as follows: under the natural light conditions, the daytime temperature was set as $25-28^{\circ} \mathrm{C}$, night temperature as $15-20^{\circ} \mathrm{C}$, and relative humidity as $65-75 \%$. After the emergence of the first true leaf, $1 / 2$ units of Yamasaki lettuce nutrient solution formula were applied. Thereafter, robust seedlings with uniform growth were selected and transplanted to hydroponic systems (Yamasaki lettuce nutrient solution; $\mathrm{pH} \approx 5.8 ; \mathrm{EC} \approx$ $1.5 \mathrm{mS} \mathrm{cm} \mathrm{cm}^{-1}$ ) with a closed LED light source in a plant factory. The environmental conditions in the plant factory were controlled as follows: daytime temperature as $24^{\circ} \mathrm{C}$, night temperature as $18^{\circ} \mathrm{C}$, humidity as $70 \pm 5 \%, \mathrm{CO}_{2}$ concentration as $400 \mu \mathrm{mol} \mathrm{mol}{ }^{-1}$, and the day/night cycle as $16 / 8 \mathrm{~h}$. The seedlings were divided into two sets, and one set was placed under white LED light and the other set was kept under the LED R and B (4:3) which was beneficial for non-heading Chinese cabbage growth for $7 \mathrm{~d}$, further named $\mathrm{W7}, \mathrm{R}$, and B7, respectively. The LED panels used here was cool white LEDs, including wavelengths from
400-800 nm. The other was a combination of R LEDs (peak at $661 \mathrm{~nm}$ ) and B LEDs (peak at $437 \mathrm{~nm}$ ). The ratio of the R:B photon flux was 4:3, which was presented as relative spectral distribution (Fig. $1 \mathrm{~S}$, supplement). All the seedlings, were subjected to $200 \pm 5 \mu \mathrm{mol}$ (photon) $\mathrm{m}^{-2} \mathrm{~s}^{-1}$ as measured by a high accuracy spectroradiometer (PARNIR, Apogee Instruments Inc., Logan, UT, USA). After the treatment, eight plants per treatment were used. The phenotypic pictures and relative physiological parameters were taken using camera and measured using $\mathrm{LI}-6400$ ( $\mathrm{Li}$ Cor, USA), and the Chl content was measured according to the method of Arnon (1949). Then, the plants were washed three times with deionized water, and leaves of $\mathrm{W} 7, \mathrm{R}$, and B7 were immediately frozen in liquid nitrogen and stored in a refrigerator at $-80^{\circ} \mathrm{C}$. All treatments were repeated three times.

RNA preparation: Total RNA was extracted using a Plant RNA Extraction Kit (TIANGEN Beijing, China) and treated with DNAase I (Zoonbio Biotechnology, China) from leaves of non-heading Chinese cabbage, which were snap-frozen and stored at $-80^{\circ} \mathrm{C}$ until processing. The RNA purity was assessed using the NanoPhotometer ${ }^{\circledR}$ spectrophotometer (IMPLEN, CA, USA). The RNA concentration was measured using a Qubit ${ }^{\circledR} R N A$ Assay Kit in a Qubit ${ }^{\circledR} 2.0$ fluorometer (Life Technologies, CA, USA). The RNA integrity was assessed using the RNA Nano 6000 Assay Kit for the Agilent Bioanalyzer 2100 system (Agilent Technologies, CA, USA).

Library preparation: A total of $3 \mu \mathrm{g}$ of RNA per sample was used as the input material for the RNA sample preparations. The sequencing libraries were generated using an NEBNext ${ }^{\circledR}$ Ultra $^{\mathrm{TM}}$ RNA Library Prep Kit for Illumina $^{\circledR}(N E B$, USA) following the manufacturer's recommendations, and index codes were added to attribute sequences to each sample. The library quality was assessed using an Agilent Bioanalyzer 2100 system.

Transcriptome sequencing: The TruSeq PE Cluster Kit v3-cBot-HS (Illumina) was used according to the manufacturer's instructions. After the cluster generation, the library preparations were sequenced on an IlluminaHiseq platform, and the paired-end reads were generated.

Quality control: Raw data in fastq format were first processed using in-house Perl scripts. During this step, clean data (clean reads) were obtained by removing reads containing adapters, reads containing poly-N and lowquality reads from the raw data. Simultaneously, the Q20, Q30, GC-content, and sequence duplication level of the clean data were calculated. All downstream analyses were based on clean, high-quality data.

Read mapping to the reference genome: The reference genome and gene model annotation files were directly downloaded from the genome website (ftp:// ftp.ncbi.nlm.nih.gov/genomes/all/GCF/000/309/985/ GCF_000309985.1_Brapa_1.0/GCF_000309985.1_ 
$A$

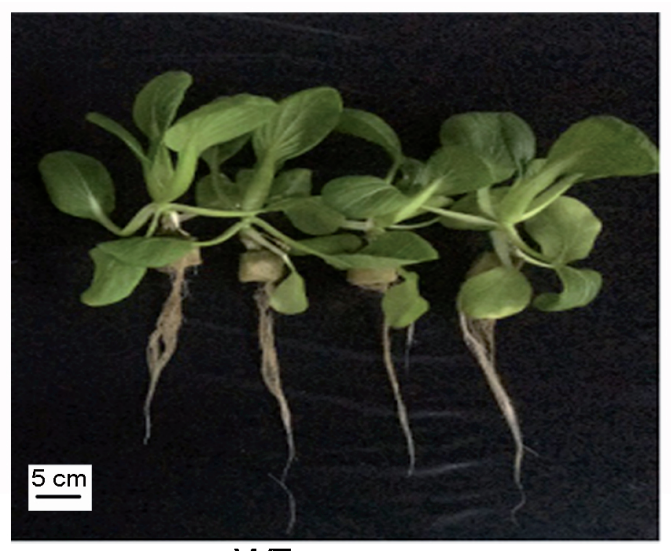

W7

$B$
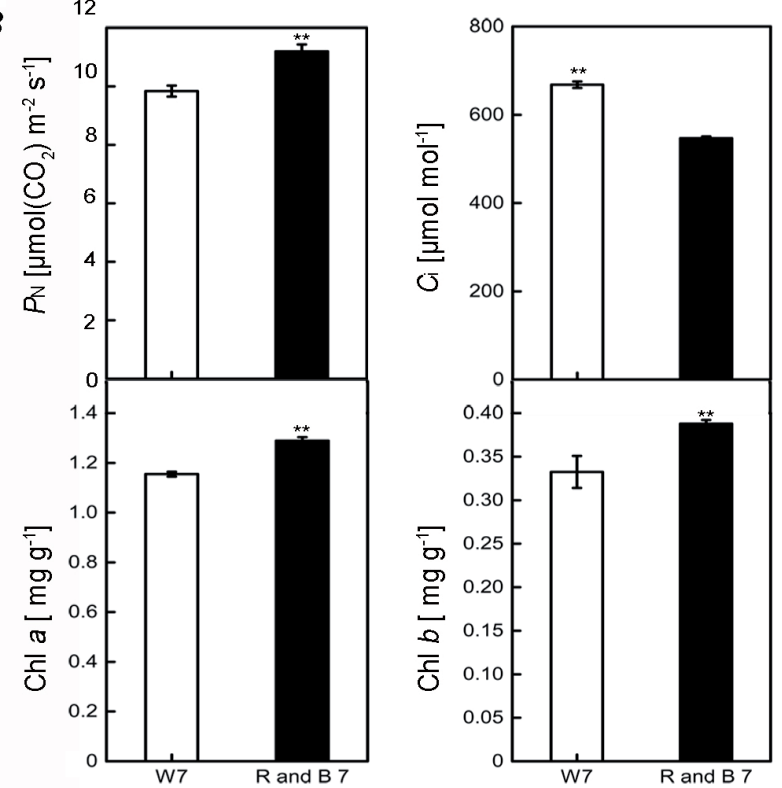

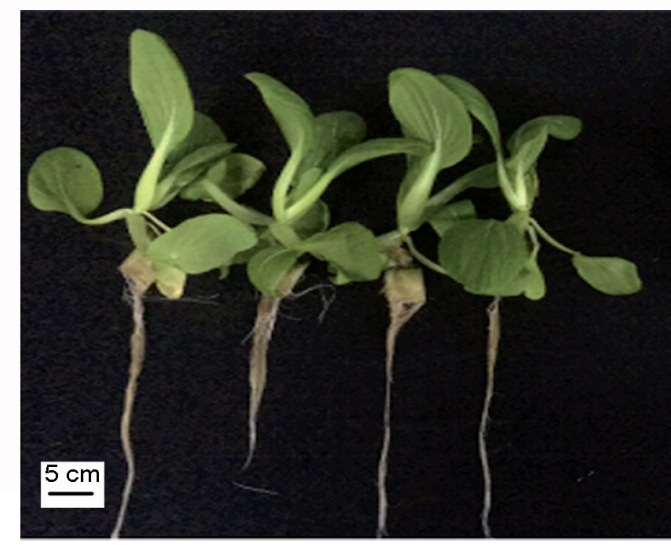

$\mathrm{R}$ and $\mathrm{B} 7$

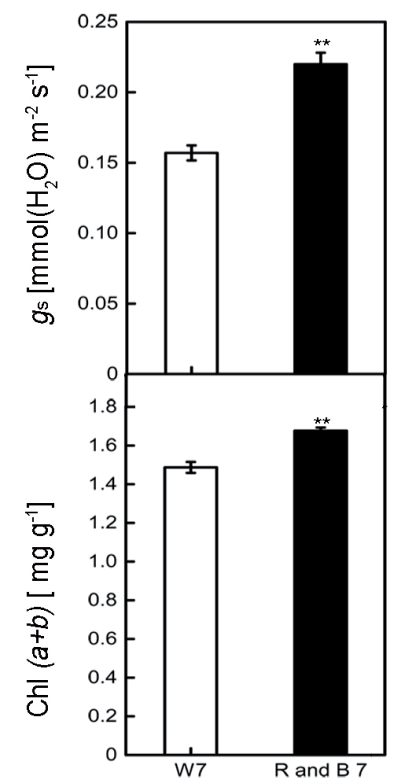

Fig. 1. The phenotype and physiological index change under $\mathrm{R}$ and $\mathrm{B}$ combination $(A)$. (B) Several physiological indexes, including intercellular $\mathrm{CO}_{2}$ concentration $\left(C_{\mathrm{i}}\right)$, net photosynthesis $\left(P_{\mathrm{N}}\right)$, stomatal conductance $\left(g_{\mathrm{s}}\right)$, transpiration rate $(E)$, and chlorophyll $(\mathrm{Chl})$ content, significantly increased under R and B treatment for 7 days. ${ }^{*},{ }^{* *}-$ significant differences at $P<0.05, P<0.01$, respectively, by Duncan's multiple comparison test.

Brapa_1.0_genomic.gbff.gz). An index of the reference genome was built, and the paired-end clean reads were aligned to the reference genome using HISAT2 (Kim et al. 2015). We selected HISAT2 as the mapping tool because HISAT2 can generate a database of splice junctions based on the gene model annotation file and, thus, provide a better mapping result than other non-splice mapping tools. Compared to TopHat, HISAT2 can produce more accurate results with greater efficiency (Kim et al. 2015).

Quantification of gene expression levels: StringTie was used to count the read numbers mapped to each gene (Pertea et al. 2016). Clean data were mapped back onto the reference genome. The read count for each gene was obtained from the mapping results, and the FPKM of each gene was calculated based on the length of the gene and reads count mapped to this gene. FPKM, which is the expected number of fragments per kilobase of transcript sequence per millions base pairs sequenced, simultaneously considers the effect of the sequencing depth and the gene length for the read counts and is currently the most commonly used method for estimating gene expression levels (Trapnell et al. 2010). To assess the variability among the samples, we performed hierarchical clustering and principal-component analysis (PCA). Hierarchical clustering was performed based on Euclidean distances. PCA was conducted using the prcomp command with the default parameters in the $R$ software package.

Differential expression analysis: The differential expression analysis between two conditions/groups was performed using the DESeq $2 R$ package (Anders and Huber 2010, Love et al. 2014). The expression levels of these genes were measured in each sample using fragments per kilobase per million reads (FPKM) values (Trapnell et al. 2010). DESeq 2 provides statistical routines for determining differential expression in digital gene expression data using a model based on a negative binomial distribution. The 
resulting $P$-values were adjusted using the Benjamini and Hochberg's approach for controlling the false discovery rate. Genes with an adjusted $P$-value $<0.05$ in DESeq 2 were considered differentially expressed.

Gene functional annotation and enrichment analysis: The gene function was annotated based on the sequence similarity in the following databases: $\mathrm{Nr}, \mathrm{Nt}, \mathrm{Pfam}, \mathrm{KOG}$ / $C O G$, Swiss-Prot, KO, and GO. The GO enrichment analysis of the DEGs was implemented using the GOseq $R$ packages based on a Wallenius noncentral hypergeometric distribution (Young et al. 2010), which can adjust for the gene length bias in the DEGs. KEGG (Kanehisa et al. 2008) is a database resource for understanding the highlevel functions and utilities of a biological system, such as a cell, organism or ecosystem, based on molecularlevel information, particularly, large-scale molecular datasets generated by genome sequencing and other high-throughput experimental technologies (http://www. genome.jp/kegg/). We used KOBAS (Mao et al. 2005) software to test the statistical enrichment of DEGs in $K E G G$ pathways.

Real-time RT-PCR analysis: To validate the accuracy of the genes obtained from the assembled transcriptome and profiling of gene expression via RNA-Seq, the expression levels of 15 selected DEGs related to photosynthesis were identified using real-time RT-PCR. Total RNA was extracted from leaves of non-heading Chinese cabbage (the same with the RNA-seq samples) using a Plant RNA Extraction Kit (Zoonbio Biotechnology, China) and purified using an RNA purification kit (Tiangen, China). The single-stranded cDNAs used for real-time PCR analysis were synthesized from the RNAs using a Prime Script II 1st Strand cDNA Synthesis Kit (TaKaRa, Dalian, China). The expression patterns of the selected DEGs were monitored. Detailed information about these DEGs is presented in Table 4S (supplement). Triplicates of each reaction were performed, and the actin (accession number: XM_024058244 gene was chosen as an internal control for normalization. The relative expression was calculated using the $2^{\Delta \mathrm{Ct}}$ method (Livaka and Schmittgen 2001). All data are expressed as the mean \pm the SD after normalization.

Data access: The raw data in this study have been deposited in NCBI's and are accessible through Bioproject

Table 1 Summary of transcriptome data. with the number of PRJNA438724.

Statistical analyses: All the data in this study were analyzed using one-way analysis of variance (ANOVA) followed by Duncan's multiple comparison test. * and $* *$ indicate significant differences at $P<0.05$ and $P<0.01$, respectively.

\section{Results}

The effects of $R$ and $B$ on plant phenotype and physiology: Compared with the white light, growth of nonheading Chinese cabbage was faster and the plant height showed a significant increase under $\mathrm{R}$ and $\mathrm{B}$ conditions, indicating that $\mathrm{R}$ and $\mathrm{B}$ efficiently promoted the growth and development of the plants. In addition, other physiological parameters, including net photosynthetic rate $\left(P_{\mathrm{N}}\right)$, stomatal conductance $\left(g_{\mathrm{s}}\right)$, transpiration rate $(E)$, and Chl content, all of them significantly increased under $\mathrm{R}$ and $\mathrm{B}$ treatment for $7 \mathrm{~d}$, indicating that the $\mathrm{R}$ and $\mathrm{B}$ combination could significantly enhance the photosynthesis of nonheading Chinese cabbage (Fig. 1).

RNA-seq results and analysis: Two non-heading Chinese cabbage sequencing data (W7, R and B7) generated from four cDNA libraries of the two conditions, i.e., control (white light) and an R and B combination (4:3) for 7 days with two biological replicates respectively, were analyzed by Illumina RNA-Seq technology. After the quality checks to remove adaptor sequences containing $\mathrm{N}$ reads and lowquality reads, in total, 29.88 gigabase $(\mathrm{Gb})$ clean read pairs were obtained from the eight RNA libraries, with an average of $7.47 \mathrm{~Gb}$ clean reads per sample (Table 1 ).

In total, $84.5 \%$ of the clean reads were mapped to the Brassica rapa reference genome, and $91.6 \%$ of the reads covered the exonic regions (Table 1). After the reconstruction of the transcripts using String Tie software, 50,190 genes were identified. The distribution and dispersion of their expression levels are shown in Fig. $2 \mathrm{~S} A, B$ (supplement), respectively, suggesting that the distribution of the gene expression was similar in all the samples.

Hierarchical clustering and a principal component analysis (PCA) were performed to assess the biological variability among the samples. Most of the variation in the gene expression was a consequence of the $\mathrm{R}$ and $\mathrm{B}$ combination treatment. The following two distinct groups were formed in the hierarchical clustering: plants treated

\begin{tabular}{llllll}
\hline & \multicolumn{2}{l}{ Clean bases [bp] GC [\%] } & $\geq \mathrm{Q} 30$ & Reads aligned & $\begin{array}{l}\text { Genes } \\
\text { covered }\end{array}$ \\
\hline White 7(W7-1) & 8056912800 & $47.11 \%$ & $91.56 \%$ & $85.36 \%$ & $92.51 \%$ \\
White 7(W7-2) & 6709419600 & $47.2 \%$ & $90.34 \%$ & $85.37 \%$ & $92.08 \%$ \\
LED R \& B 7(R and B7-1) & 8591208000 & $46.69 \%$ & $91.36 \%$ & $83.37 \%$ & $91.56 \%$ \\
LED R \& B 7(R and B7-2) & 6527319600 & $46.54 \%$ & $90.26 \%$ & $83.93 \%$ & $90.03 \%$ \\
Total & 29884860000 & & & & \\
Average & 7471215000 & & & $84.52 \%$ & $91.55 \%$ \\
\hline
\end{tabular}


with the $\mathrm{R}$ and $\mathrm{B}$ combination and plants treated with white light (Fig. 3S, supplement).

Identification, functional annotation, and characterization of differentially expressed genes (DEGs): In total, 5,643 genes showed significant variations in expression according to the DESeq analysis as follows: 2,893 genes were upregulated, and 2,750 genes were downregulated (Fold Change $\geq 2$ and FDR $<0.05$ ) in the non-heading Chinese cabbage plants under the $\mathrm{R}$ and $\mathrm{B}$ combination compared with the white light (Fig. $2 A, 1 \mathrm{~S}$ - supplement) and PCA showed that the transcriptomes of the two groups clearly differed (Fig. $2 B$ ). The functional annotation of the 5,643 DEGs revealed that 5,363, $3,959,3,743,1,224,4,905,2,625$, and 1,736 DEGs had alignments to the Non-redundant protein database (NR), Annotated protein sequence database (Swiss Prot), Protein family database (Pfam), Kyoto encyclopedia of genes and genomes database (KEGG), Gene Ontology database (GO), euKaryoticOrtholog Groups database (KOG), and Clusters of orthologous groups of proteins database (COG), respectively. $G O$ analysis showed that in the cell components, DGEs were mainly enriched in cell, cell part and organelle; in the molecular function, DGEs were mainly enriched in binding, catalytic activity, and in the biological process, DGEs were mainly involved in cellular processes, metabolic processes, single organism processes (Fig. $4 \mathrm{~S}$ - supplement). The KEGG enrichment analysis showed that the annotated genes involved in plant hormone signal transduction (86), ribosome (55), plantpathogen interaction (48), carbon fixation in photosynthetic organism (40), and phenylalanine biosynthesis (38) (Fig. $3 A$ ). The top 20 pathways with the most significant enrichment level was photosynthesis - antenna proteins, followed by photosynthesis, carbon fixation in photosynthetic organisms, Car biosynthesis, and porphyrin and Chl metabolism (Fig. 3B), suggesting that the $\mathrm{R}$ and $\mathrm{B}$ combination might regulate the growth and development of non-heading Chinese cabbage by regulating photosynthesis and the biosynthesis of Chl and Car compared with white light.

$\mathbf{R}$ and $\mathbf{B}$ combination regulated photosynthesis: In total, 127 DEGs related to photosynthesis were regulated in the non-heading Chinese cabbage under the $\mathrm{R}$ and $\mathrm{B}$ combination compared with white light; of these DEGs, 33 were enriched in the photosynthetic pathway in the KEGG database (Fig. 3; Table 1S, supplement). One gene encoding the PsaH2 subunit of PSI was downregulated, and 14 genes encoding subunits of PSII, including one PsbP, six PsbQs, two PsbRs, two PsbYs, one Psb27, and two Psb28s, were nonregulated. Meanwhile, four genes encoding the cytochrome $b_{6} f$ complex subunits (one PetD and three PetCs), eight genes involved in the photosynthetic electron transport (four PetFs, three PetHs, one cytco) and six ATP synthase family genes were upregulated.

$\mathbf{R}$ and $\mathbf{B}$ combination regulated antenna proteins: Forty genes encoding antenna proteins were identified in nonheading Chinese cabbage under the $\mathrm{R}$ and $\mathrm{B}$ combination; 22 of these genes were enriched in photosynthesis antenna proteins in the $K E G G$ database. Two genes encoding antenna proteins in PSI, i.e., Lhca2 and Lhca5, were upregulated, and three genes encoding antenna proteins in PSI, i.e., Lhcal, Lhca3, and Lhca4, were
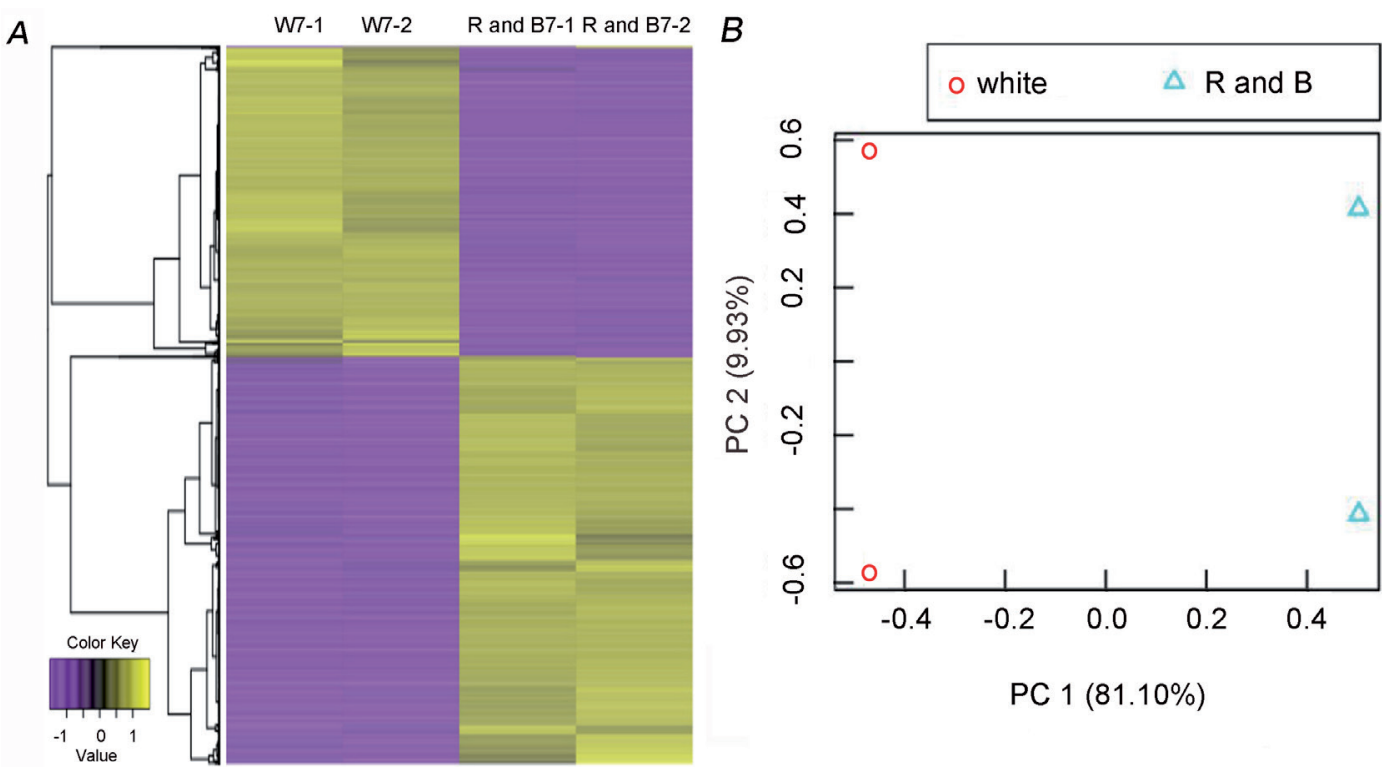

Fig. 2. Heat map of all up- and downregulated genes among 4 samples. Colors bar represented expression levels of each gene which were either upregulated (yellow) or downregulated (purple). (A) Compared with the white light, in total 5,643 genes showed significant variations in expression according to the DESeq analysis, among of which 2,893 genes were upregulated and 2,750 genes were downregulated under the standard of Fold Change $\geq 2$ and FDR $<0.05$ in the non-heading Chinese cabbage plants under the R and B combination. (B) PCA analysis showed that the transcriptomes of the two groups clearly differed. 
A
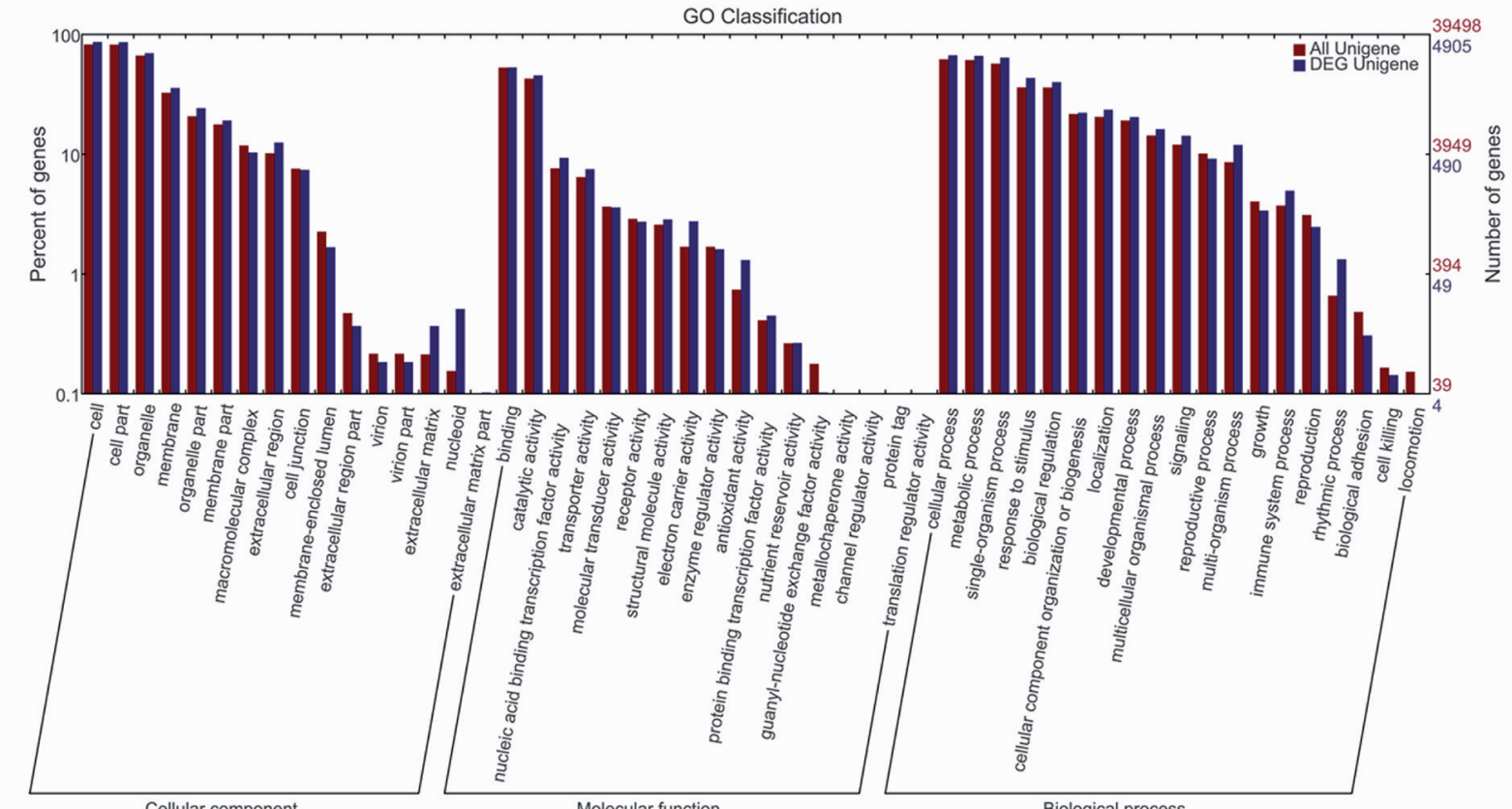

$B$
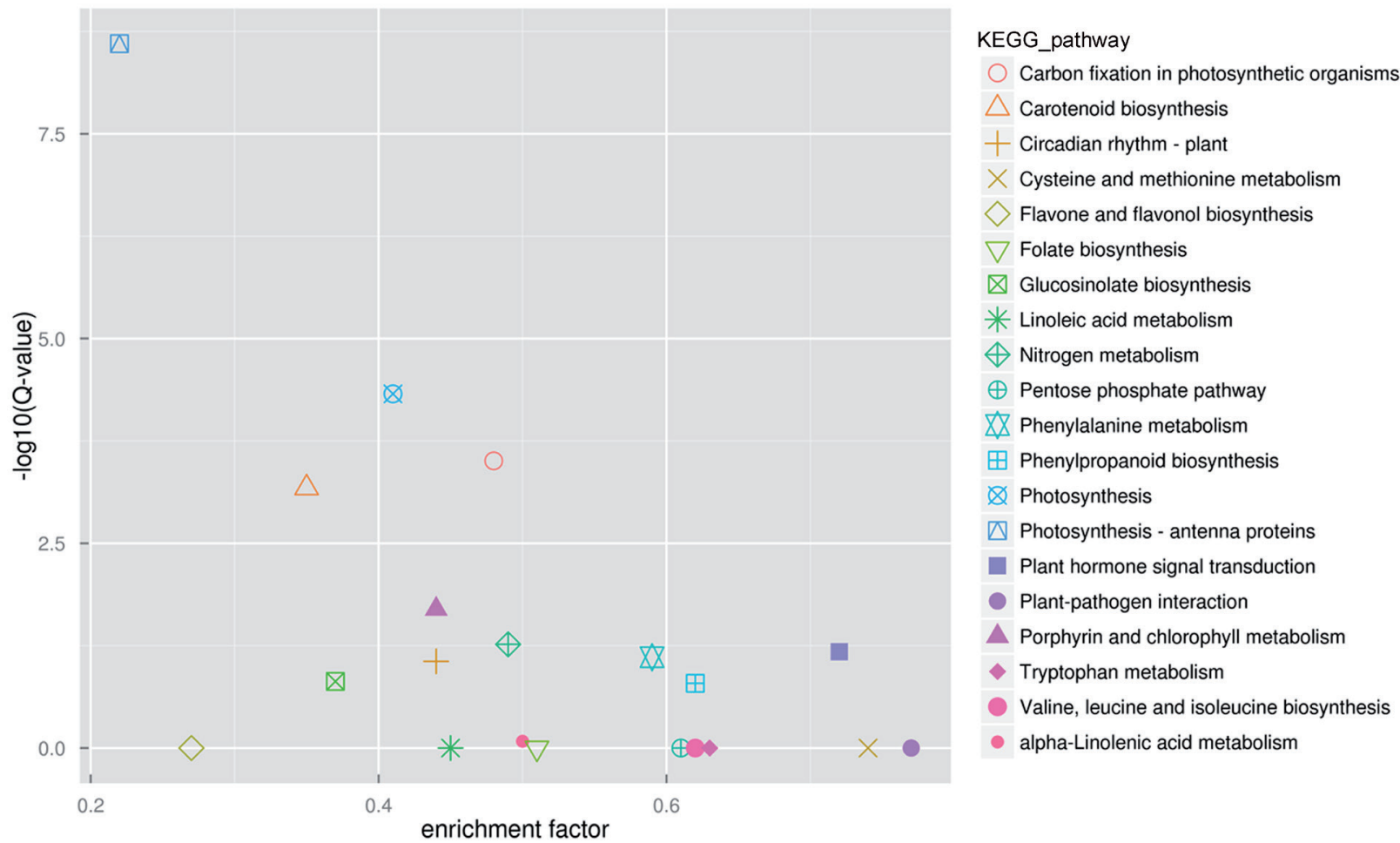

Fig. 3. KEGG analysis of annotated genes. Pathway prediction of up- and down-regulation DEGs between W7 and R and B 7. ( $A$ ) The $K E G G$ enrichment analysis showed that the pathways of top five contained plant hormone signal transduction, ribosome, plant-pathogen interaction, carbon fixation in photosynthetic organism, and phenylalanine biosynthesis, respectively. $(B)$ The top 20 pathways with the most significant enrichment level was photosynthetic antenna proteins, followed by photosynthesis, carbon fixation in photosynthetic organisms, Car biosynthesis and porphyrin, and Chl metabolism.

downregulated. Six genes encoding Lhcb1s and four genes encoding $L h c b 2 s$ in PSII were upregulated, and seven genes encoding $L h c b$ s in PSII, including two Lhcb3s, two Lhcb4s, and three Lhcb6s, were downregulated. These results suggested that the $\mathrm{R}$ and $\mathrm{B}$ combination increased the expression levels of antenna proteins.
$R$ and $B$ combination regulated $\mathrm{Chl}$ biosynthesis: More than 200 genes involved in Chl biosynthesis were identified in non-heading Chinese cabbage under the $\mathrm{R}$ and $\mathrm{B}$ combination, and 18 of these genes were enriched in the Chl biosynthesis pathway in the $K E G G$ database (Fig. 4; Table 2S, supplement). 
Twelve genes encoding eight key enzymes in the Chl biosynthesis pathway were upregulated under the $\mathrm{R}$ and B combination compared with white light (Fig. 5, Table 2S). One of these genes encoding glutamyltRNAreductase $(\operatorname{Hem} A)$, which catalyzes L-glutamyltRNA conversion into L-glutamate-1-semialdehyde.This step precedes 5-aminolevulinate formation, which is the rate-limiting step in Chl biosynthesis. Two genes encoding porphobilinogendeaminase $(\mathrm{HemC})$, which convert porphobilinogen to hydroxymethylbilane. Two genes encoding coproporphyrinogen-III oxidase 1 (HemF), and one gene encoding S-adenosylmethionine (HemN). $H e m F$ and $H e m N$ jointly catalyze the formation of protoporphyrinogen IV from coproporphyrinogen-III. Two genes encoding magnesium-chelatasesubunit 1 (ChlI1) and magnesium-chelatase subunit 2 (ChlI2), one gene encoding magnesium protoporphyrin-IX methyltransferase $(C h l M)$ and two genes encoding magnesium protoporphyrin-IX monomethyl ester cyclases (CRD1s) jointly catalyze the conversion of protoporphyrin IV to divinyl-protochlorophyllide through $\mathrm{Mg}$-protoporphyrin Photosystem II

D1 $\quad$ D2
\begin{tabular}{|l|l|l|l|l|l|l|l|}
\hline PsbA & PsbD & PsbC & PsbB & PsbE & PsbF & \multicolumn{1}{c|}{ MSP } & OEC \\
\hline PsbL & PsbJ & PsbK & PsbM & PsbH & Psbl & PsbO & PsbP \\
\hline PsbQ & PsbR & PsbS & PsbT & PsbU & PsbV & PsbW & PsbX \\
\hline PsbY & PsbZ & Psb27 & Psb28 & Psb28-2 & & & \\
\hline
\end{tabular}

Photosystem I
\begin{tabular}{|l|l|l|l|l|l|l|l|}
\hline PsaA & PsaB & PsaC & PsaD & PsaE & PsaF & PsaG & PsaH \\
\hline Psal & PsaJ & PsaK & PsaL & PsaM & PsaN & PsaO & PsaX \\
\hline
\end{tabular}

IV monomethyl ester and magnesium protoporphyrin IV. One gene, encoding Chl synthase $(C h l G)$, catalyzes the conversion of chlorophyllide $a / b$ to $\mathrm{Chl} a / b$, which is the final step of Chl biosynthesis. One gene encoding chlorophyllase $(D H O D)$, which catalyzes the conversion of Chl $a / b$ into chlorophyllide $a / b$, was downregulated under the $\mathrm{R}$ and $\mathrm{B}$ combination and enhanced $\mathrm{Chl}$ biosynthesis. These results showed that more than half of the genes involved in the $\mathrm{Chl}$ biosynthesis pathway were upregulated in non-heading Chinese cabbage under the $\mathrm{R}$ and $\mathrm{B}$ combination, providing a solid foundation for the promotion of photosynthesis, and were consistent with the increase in the Chl content that has been observed in previous studies (Kim et al. 2004, Wang et al. 2009, 2015, 2016; Hogewoning et al. 2010).

Notably, one gene encoding chlorophyll deoxygenase $(C A O)$ was downregulated, and the expression patterns of three genes encoding protochlorophyll dereductases $(P O R s)$ differed in non-heading Chinese cabbage under the $\mathrm{R}$ and $\mathrm{B}$ combination.

\begin{tabular}{|c|c|c|c|c|c|c|c|}
\hline \\
\hline PetB & PetD & PetA & PetC & PetL & PetM & PetN & PetG \\
\hline \multicolumn{8}{|c|}{ Photosynthethic electron transport } \\
\hline PC & $\mathrm{Fd}$ & FNR & cytc & & & & \\
\hline PetE & PetF & PetH & PetJ & & & & \\
\hline
\end{tabular}

F-type ATPase

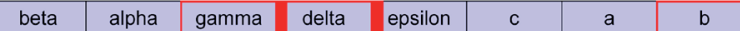

Fig. 4. The DEGs enriched in photosynthesis pathway in Brassica campestris L. under R and B combination and their locations in the pathway. Compared with the white light, in total, 127 DEGs related to photosynthesis were regulated in the non-heading Chinese cabbage under the R and B combination, among of these DEGs, 33 were enriched in the photosynthesis pathway in the $K E G G$ database. Among of these genes, a gene encoding the PsaH2 subunit of PSI was downregulated, and 14 genes encoding subunits of PSII, including one PsbP, six PsbQs, two PsbRs, two PsbYs, one Psb27, and two Psb28s, were nonregulated. Meanwhile, four genes encoding the cytochrome $b_{6} f$ complex subunits, eight genes involved in the photosynthetic electron transport, and six ATP synthase family genes were upregulated.

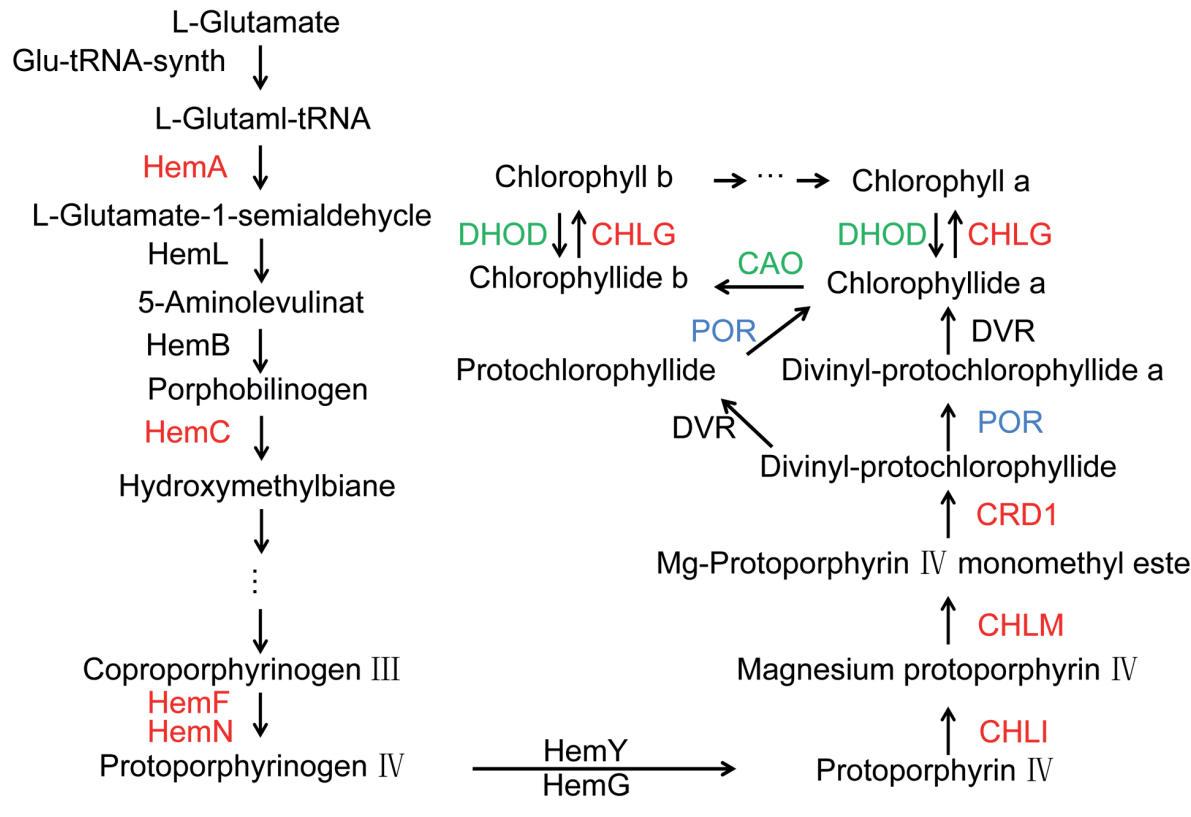

Fig. 5. The DEGs enriched in Chl biosynthesis pathway in Brassica campestris $\mathrm{L}$. under $\mathrm{R}$ and $\mathrm{B}$ combination and their locations in the pathway. Red and green color represents upregulated and downregulated, respectively. Blue color represents some genes encoding a protein upregulated and the others downregulated simultaneously. Compared with the white light, more than 200 genes involved in $\mathrm{Chl}$ biosynthesis have been identified in non-heading Chinese cabbage under the $\mathrm{R}$ and $\mathrm{B}$ combination, and 18 of these genes were enriched in the Chl biosynthesis pathway in the $K E G G$ database. Moreover, 12 genes encoding eight key enzymes in the $\mathrm{Chl}$ biosynthesis pathway were upregulated under the $\mathrm{R}$ and $\mathrm{B}$ combination compared with white light. 
$\mathbf{R}$ and $\mathbf{B}$ combination regulated Car biosynthesis: Fifty three genes involved in Car biosynthesis were identified in non-heading Chinese cabbage under the $\mathrm{R}$ and $\mathrm{B}$ combination, and 20 of these genes were enriched in the Car biosynthesis pathway in the $K E G G$ database compared with the white light (Fig. 6; Table 3S, supplement). The genes, which encode 10 proteins that play roles in Car biosynthesis, were upregulated, including two genes that encodes geranylgeranyl-diphosphate geranylgeranyl transferases or phytoene synthases (PSYS), phytoenedesaturase $(P D S 3), \zeta$-carotene desaturase $(Z D S)$, prolycopeneisomerase $(\mathrm{CrtH})$, lycopene cyclase $(\mathrm{CruA})$, lycopene $\varepsilon$-cyclase $(C r t L-e)$, carotene $\varepsilon$-monooxygenase $(L U T 1)$, zeaxanthinepoxidase $(Z E P)$, violaxanthin deepoxidase $(V D E)$, and three genes that encode abscisic acid 8'-hydroxylases $(C Y P S)$. The genes encoding the three proteins were downregulated, including one gene encoding $\beta$-carotene 3-hydroxylase 1 ( $C r t Z)$, four genes encoding 9-cis-epoxycarotenoid dioxygenases $(N C E D s)$, and three genes encoding xanthoxin dehydrogenases $(A B A 2 s)$.

PSY catalyzes the phytoene formation from all-transgeranylgeranyl-PP, which is an intermediate step in the biosynthesis of terpenes and terpenoids. PDS3 catalyzes the conversion of phytoene into phytofluene and then into $\zeta$-carotene. ZDS and $\mathrm{CrtH}$ jointly catalyze the conversion of $\zeta$-carotene into lycopene though neurosporene. Lycopene is successively converted into $\gamma$-carotene and $\beta$-carotene by CruA and then into $\delta$-carotene and $\varepsilon$-carotene by CrtL-e. The increase in the expression levels of these genes suggested an increase in some types of Car, such as phytofluene, $\zeta$-carotene, lycopene, $\gamma$-carotene, $\beta$-carotene, $\delta$-carotene, $\varepsilon$-carotene, $\alpha$-carotene, echinenone, and canthaxanthin under the $\mathrm{R}$ and $\mathrm{B}$ combination, which is consistent with the increased Car contents observed in previous studies (Kim et al. 2004, Wang et al. 2009, 2015, 2016; Hogewoning et al. 2010).

Lutein is formed from $\alpha$-carotene by two catalysis steps that are mediated by LUT1 and CrtZ. Although LUT1 was upregulated in this study, the $\mathrm{R}$ and $\mathrm{B}$ combination might not increase the lutein content in non-heading Chinese cabbage because of the downregulated Crt $Z$. Similar results were observed in the formation of other carotenes and intermediates, including $\beta$-cryptoxanthin, 3-hydroxyechinenone, phoenicoxanthin, adonixanthin, and astaxanthin, which were all formed by CrtZ catalysis.

The xanthophyll cycle protects the photosynthetic apparatus from photodamage caused by light-induced oxidative stress in photosynthetic organisms. In higher plants, ZEP and $V D E$ catalyze the conversion among three Car pigments, i.e., violaxanthin, antheraxanthin, and zeaxanthin, under low-light conditions and high-light conditions, respectively. These enzymatic cycles play a key role in stimulating energy dissipation within the light-harvesting antenna proteins by nonphotochemical quenching, which is a major method of protecting against photoinhibition (Latowski et al. 2011). The upregulation of $Z E P$ and $V D E$ suggested that the $\mathrm{R}$ and $\mathrm{B}$ combination enhanced photodamage tolerance in non-heading Chinese

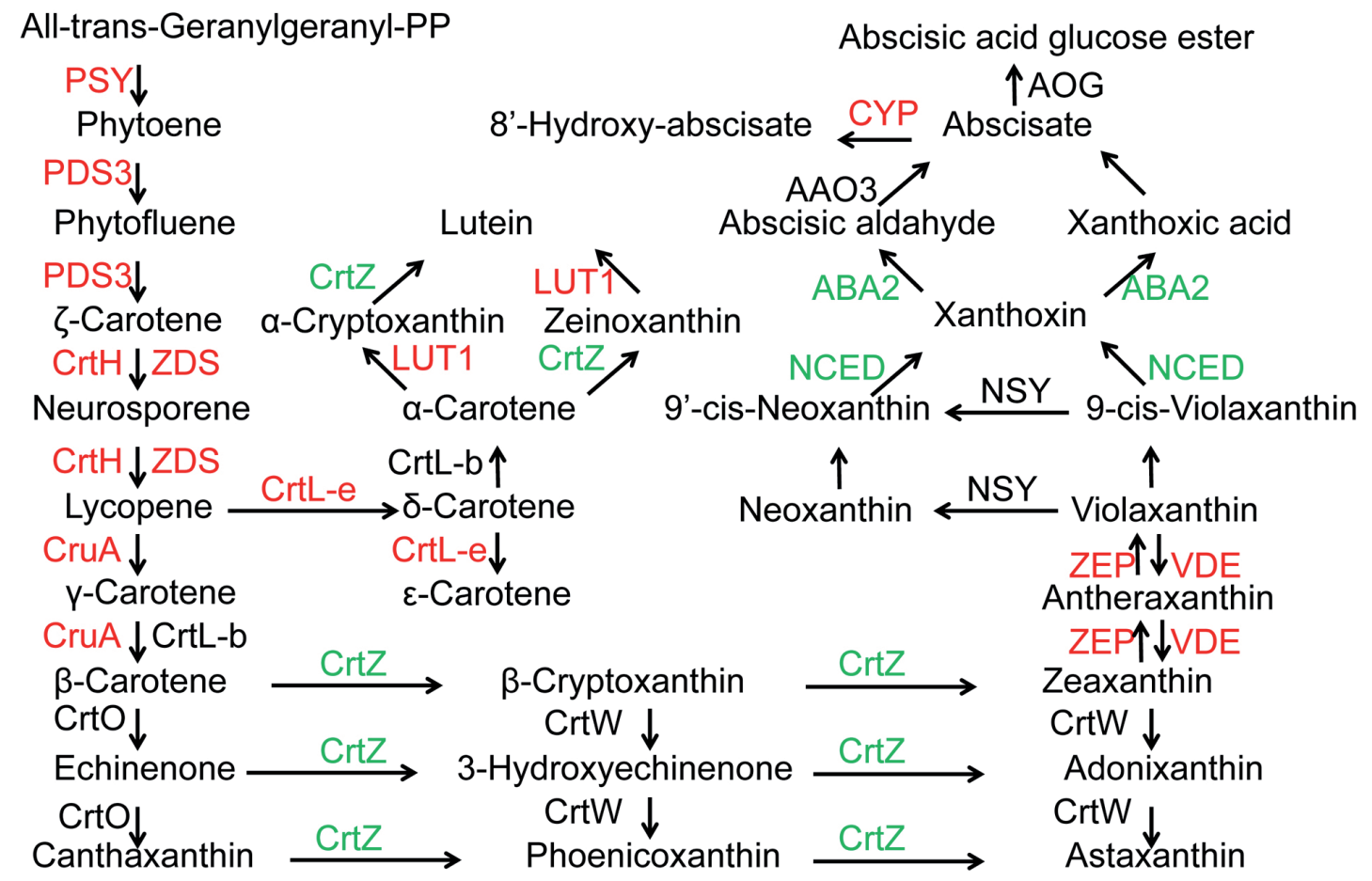

Fig. 6. The DEGs enriched in Car biosynthesis pathway in Brassica campestris L. under R and B combination and their locations in the pathway. Red and green color represents upregulated and downregulated, respectively. Compared with the white light, 53 genes involved in carotenoid biosynthesis were identified in non-heading Chinese cabbage under the R and B combination, and 20 of these genes were enriched in the Car biosynthesis pathway in the KEGG database. Moreover, NCED, ABA2 and CYP were involved in the abscisic acid biosynthesis. The genes of $N C E D$ and $A B A 2$ were downregulated, and $C Y P$ was upregulated under the $\mathrm{R}$ and $\mathrm{B}$ combination. 
cabbage.

NCED, ABA2, and CYP were involved in the abscisic acid biosynthesis. The genes of NCED and $A B A 2$ were downregulated, and $C Y P$ was upregulated under the $\mathrm{R}$ and B combination (Fig. 6 and Table 3S), suggesting that ABA biosynthesis might be depressed by the $\mathrm{R}$ and $\mathrm{B}$ combination.

Real-time PCR verification: To validate the gene expression change obtained by RNA-Seq under white light (W7) and red and blue combination (R and B7) for $7 \mathrm{~d}$, 15 DEGs, including HemA, PORC, PsbQ, PetC, FNR1, ATPC1, Lhca5, CrtZ, ZEP, NCED1, NCED2, CRD1, $H E M C, P e t D$, and PsbY were selected for real-time PCR analysis. The results indicated that 13 of 15 genes showed the similar expression patterns with the RNA-seq data except for $C R D 1$ and $H E M C$, confirming the reliability and accuracy of the RNA-Seq data in this study (Fig. 7 and Tables 4S, 5S - supplements).

\section{Discussion}

Plants obtain energy from light for growth and development through photosynthesis. $\mathrm{R}$ and $\mathrm{B}$, which are the two
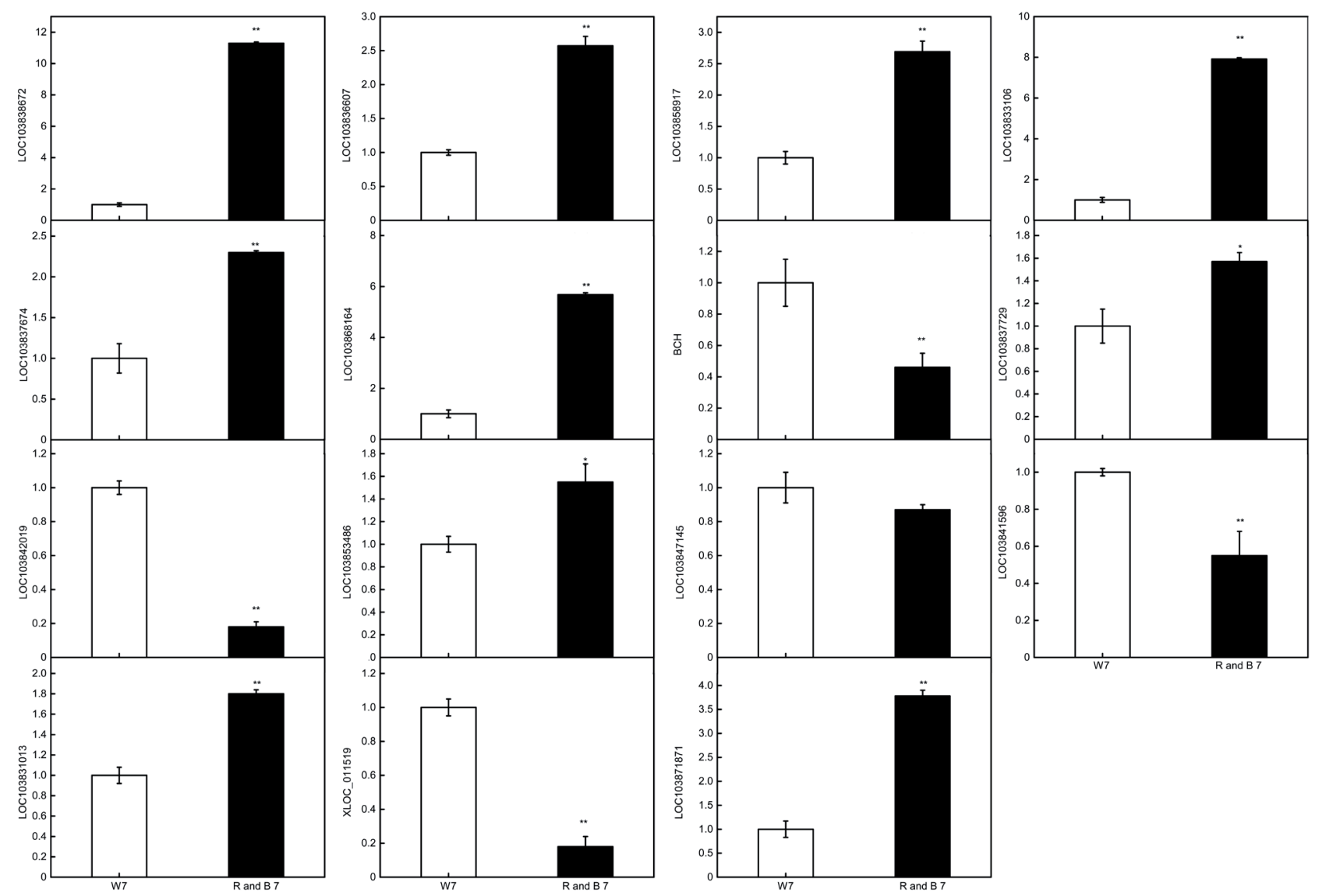

Fig. 7. RT-qPCR analyses of 15 DGEs under white light (W7) and red and blue combination (R and B7) for 7 days. Each bar represents the mean \pm STD of triplicate assays. Asterisks indicate significant differences between white light (control value $=1$ ) and LED red and blue combination, * and ** indicate significant differences at $P<0.05$ and $P<0.01$, respectively by Duncan's multiple comparison test. In total, 15 DEGs, including HemA, PORC, PsbQ, PetC, FNR1, ATPC1, Lhca5, CrtZ, ZEP, NCED1, NCED2, CRD1, HEMC, PetD, and $P s b Y$ were selected for real-time PCR analysis, and 13 of 15 genes showed the similar expression patterns with the RNA-seq data except for $C R D 1$ and $H E M C$. 
in PSII and key genes in Chl and Car biosynthesis were also upregulated by the $\mathrm{R}$ and $\mathrm{B}$ combination to provide necessary support for the promoted photosynthesis (Fig. 4). In Arabidopsis treated with R for $24 \mathrm{~h}$, the greening of the etiolated seedlings occurred rapidly and correlated with a massive upregulation of more than 60 transcripts encoding photosynthesis-related proteins, including 8 PSI subunits, 5 PSII subunits, 6 LHCAs, 3 LHCBs, the minor antenna proteins CP24, CP26, and CP29, 3 LILs, 3 ATPase subunits, Fd, Fd-NADP reductase, proteins involved in Chl synthesis, and enzymes involved in the Calvin-Benson cycle, including Rubisco (Ghassemian et al. 2006). These results are consistent with our findings. In addition, many genes involved in the Calvin-Benson cycle were also upregulated by the $\mathrm{R}$ and $\mathrm{B}$ combination in our study.

Oxygenic photosynthesis is catalyzed by the following four multi-subunit membrane-protein complexes: PSI, PSII, the cytochrome $b_{6} f$ complex, and F-ATPase. PSI generates the most negative redox potential in nature and largely determines the global amount of enthalpy in living systems. PSII generates an oxidant whose redox potential is high enough to oxidize $\mathrm{H}_{2} \mathrm{O}$, which is a highly abundant substrate that ensures a practically unlimited electron source for life on Earth. PsaA-PsaL and the lightharvesting Chl-binding proteins $\mathrm{LHCa} 1, \mathrm{LHCa} 2, \mathrm{LHCa} 3$, and LHCa4 are genuine subunits of the plant PSI. The subunit composition of PSII is much more complex than that of PSI. These biochemical results define the major intrinsic proteins of eukaryotic PSII as Psb A, B, C, D, E, F, O, P, Q, and S, the polypeptides that donate axial His ligands to the heme iron of cytochrome $b_{559}$. Of the other polypeptides (Psb H-L, N, R, T, and W-Z) associated with PSII, the functions of PsbN and PsbR remain unclear (Nelson and Yocum 2006). Based on our data, the subunit of PSI was downregulated, and 14 genes encoding subunits of PSII were nonregulated, suggesting that the $\mathrm{R}$ and $\mathrm{B}$ combination might promote plant photosynthesis by regulating PSII (Fig. 4S, Table $1 \mathrm{~S}$ - supplements). Meanwhile, the subunits of the cytochrome $b_{6} f$ complex, genes involved in the photosynthetic electron transport and genes from the ATP synthase family were upregulated. These results confirmed that the $\mathrm{R}$ and $\mathrm{B}$ combination stimulated plant photosynthesis and promoted the growth and development of plants, such as the increase in $P_{\mathrm{N}}$, $P_{\max }, \mathrm{LMA}$, and Chl content (Kim et al. 2004, Wang et al. 2009, 2015, 2016; Hogewoning et al. 2010).

The light-harvesting complex, which is also called the antenna complex, is an array of antenna proteins and Chl or Car molecules embedded in the thylakoid membrane of plants that is responsible for the absorption and conversion of light energy at the reaction center of a photosystem in oxygen-evolving photosynthetic organisms (Grossman et al. 1995, Green and Durnford 1996, Vasil'ev and Bruce 2004, Nelson and Yocum 2006, Ahn et al. 2008). The primary photochemical reactions in photosynthesis occur in the photosynthetic reaction centers, but their fluent and efficient operation is maintained by the activity of numerous specialized light-harvesting complexes that absorb light quanta and transfer electronic excitations to the reaction centers. Another purpose of the regulation of light-harvesting complexes in plants is to adjust the number of electronic excitations in the photosynthetic apparatus to the current capacity of the reaction centers, and light-harvesting complexes in plants are composed of antenna proteins and antenna pigments. PSI and PSII have their own antenna complexes (Grossman et al. 1995, Green and Durnford 1996, Vasil'ev and Bruce 2004, Nelson and Yocum 2006, Ahn et al. 2008). Based on our data, 11 antenna proteins were upregulated in non-heading Chinese cabbage under the $\mathrm{R}$ and $\mathrm{B}$ combination to absorb and convert more energy for promoting photosynthesis. Nine of these proteins belong to PSII, and only two belong to PSI, further demonstrating that the stimulation of photosynthesis under the $\mathrm{R}$ and $\mathrm{B}$ combination may be mediated primarily by PSII.

$\mathrm{Chl}$, which is another constituent of the light-harvesting complex, is essential for photosynthesis and enables plants to absorb energy from light. Chl biosynthesis, which begins with L-glutamate, has been well studied (Bollivar 2006, Reinbothe 2010, Stenbaek and Jensen 2010, Wang and Grimm 2015). Car, similarly as Chl, function as antenna pigments that absorb light energy for use in plant photosystems (Armstrong and Hearst 1996). The antenna pigments are predominantly $\mathrm{Chl}$ and Car. The most effective absorption of Chl occurs in the blue region of the electromagnetic spectrum, and the second most effective absorption occurs in the red region (Chen 2014). Car are organic pigments that are produced by plants, algae, several bacteria, and fungi. There are over 600 known Cars split into two classes, i.e., the xanthophylls (which contain oxygen) and carotenes (which are purely hydrocarbons and contain no oxygen). The Car that contain unsubstituted $\beta$-ionone rings (including $\beta$-carotene, $\alpha$-carotene, $\beta$-cryptoxanthin, and $\gamma$-carotene) have vitamin A activity, and these and other Car can also act as antioxidants. In general, Car absorb wavelengths ranging from $400-550 \mathrm{~nm}$ (violet to green light), which is distinct from Chl. Another key role of Car in plants and algae is to protect $\mathrm{Chl}$ from photodamage (Armstrong and Hearst 1996). In this study, 21 and 20 DEGs enriched in the Chl and Car biosynthesis pathway were identified in the $K E G G$ database, respectively, under the $\mathrm{R}$ and $\mathrm{B}$ combination. Among these DEGs, 17 and 13 DEGs were upregulated, suggesting that non-heading Chinese cabbage produced more $\mathrm{Chl}$ and Car to interact with antenna proteins, providing the necessary support for the promotion of photosynthesis, growth and development in non-heading Chinese cabbage.

Two types of $\mathrm{Chl}$ are present in the photosystems of green plants as follows: Chl $a$ and $b$. CAO converts chlorophyllide $a$ to chlorophyllide $b$. This reaction results in increased Chl $a$ and a reduction in Chl $b$ (Eggink et al. 2004). The CAO gene was downregulated (Fig. 5, Table $2 \mathrm{~S}$ ), suggesting that the $\mathrm{R}$ and $\mathrm{B}$ combination may primarily induce an increase in $\mathrm{Chl} a$, but not $\mathrm{Chl} b$, in non-heading Chinese cabbage. The downregulation of $C r t Z$ may induce the reduction of many types of Car. These data suggest that the regulation of $\mathrm{Chl}$ and carotene biosynthesis by the $\mathrm{R}$ and $\mathrm{B}$ combination was selective.

POR is responsible for the conversion of protochlorophyllide and divinyl-protochlorophyllide into 
chlorophyllide and divinyl-protochlorophyllide, respectively (Rowe and Griffiths 1995, Heyes et al. 2012). There are different POR isoforms in green plants (Sytina et al. 2008). The two isoforms, POR B and C, were differentially expressed in this study. Two $P O R B$ genes were downregulated, and two PORC genes were upregulated, suggesting that the $\mathrm{R}$ and $\mathrm{B}$ combination might specifically induce different types of PORs in the pathway in greengrocery plants.

The cross-talk between light- and ABA-signaling pathways has been demonstrated by many researchers (Galvez-Valdivieso et al. 2009, Hayashi and Kinoshita 2011, Arve et al. 2013, Xu et al. 2014). The change in the expression levels of the three genes related to ABA biosynthesis in this study proved that the $\mathrm{R}$ and $\mathrm{B}$ combination might induce the biosynthesis and regulation of plant hormones in non-heading Chinese cabbage and, thus, regulate plant growth and development. Taken together, enhanced transcript abundances allow increased plant performance under the applied red/blue light regime.

\section{References}

Ahn T.K., Avenson T.J., Ballottari M. et al.: Architecture of a charge-transfer state regulating light harvesting in a plant antenna protein. - Science 320: 794-797, 2008.

Acock B., Charles-Edwards D.A., Fitter D.J. et al.: The contribution of leaves from different levels within a tomato crop to canopy net photosynthesis: an experimental examination of two canopy models. - J. Exp. Bot. 29: 815$827,1978$.

Anders S., Huber W.: Differential expression analysis for sequence count data. - Genome Biol. 11: R106, 2010.

Armstrong G.A., Hearst J.E.: Carotenoids 2: Genetics and molecular biology of carotenoid pigment biosynthesis. FASEB J. 10: 228-237, 1996.

Aksenova N.P., Konstantinova T.N., Sergeeva L.I. et al.: Morphogenesis of potato plants in vitro. I. Effect of light quality and hormones. - J. Plant Growth Regul. 13: 143, 1994.

Arnon D.I.: Copper enzymes in isolated chloroplasts: Polyphenoloxidase in Beta vulgaris. - Plant Physiol. 24:1-15, 1949.

Appelgren M.: Effects of light quality on stem elongation of Pelargonium in vitro. - Sci. Hortic.-Amsterdam 45: 345-351, 1991.

Arve L.E., Terfa M.T., Gislerød H.R. et al.: High relative air humidity and continuous light reduce stomata functionality by affecting the ABA regulation in rose leaves. - Plant Cell Environ. 36: 382-392, 2013.

Bollivar D.W.: Recent advances in chlorophyll biosynthesis. Photosynth. Res. 90: 173-194, 2006.

Caffarri S., Tibiletti T., Jennings R.C. et al.: A comparison between plant photosystem I and photosystem II architecture and functioning. - Curr. Protein Pept. Sci. 15: 296-331, 2014.

Chen M.: Chlorophyll modifications and their spectral extension in oxygenic photosynthesis. - Annu. Rev. Biochem. 83: 317340,2014

Christie J.M.: Phototropin blue-light receptors. - Annu. Rev. Plant Biol. 58: 21-45, 2007.

Corré W.J.: Growth and morphogenesis of sun and shade plants. I. The influence of light intensity. - Acta Bot. Neerl. 32: 4962, 1983.

Cosgrove D.J.: Photomodulation of growth. - In: Kendrick R.E., Kronenberg G.H.M. (ed.): Photomorphogenesis in Plants. Pp.
341-366. Dr W. Junk Publ., Dordrecht 1986.

Eberhard S., Finazzi G., Wollman F.A.: The dynamics of photosynthesis. - Annu. Rev. Genet. 42: 463-515, 2008.

Eggink L., LoBrutto R., Brune D.C. et al.: Synthesis of chlorophyll $b$ : Localization of chlorophyllide a oxygenase and discovery of a stable radical in the catalytic subunit. - BMC Plant Biol. 4: 5, 2004.

Galvez-Valdivieso G., Fryer M.J., Lawson T. et al.: The high light response in Arabidopsis involves ABA signaling between vascular and bundle sheath cells. - Plant Cell 21: 2143-2162, 2009.

Ghassemian M., Lutes J., Tepperman J. et al.: Integrative analysis of transcript and metabolite profiling data sets to evaluate the regulation of biochemical pathways during photomorphogenesis. - Arch. Biochem. Biophys. 448: 45-59, 2006.

Green B.R., Durnford D.G.: The chlorophyll-carotenoid proteins of oxygenic photosynthesis. - Annu. Rev. Plant Phys. 47: 685-714, 1996.

Grossman A.R., Bhaya D., Apt K.E. et al.: Light-harvesting complexes in oxygenic photosynthesis: diversity, control, and evolution. - Annu. Rev. Genet. 29: 231-288, 1995.

Hayashi M., Kinoshita T.: Crosstalk between blue-light- and ABA-signaling pathways in stomatal guard cells. - Plant Signal Behav. 6: 1662-1664, 2011.

Hernández R., Kubota C.: Physiological responses of cucumber seedlings under different blue and red photon flux ratios using LEDs. - Environ. Exp. Bot. 121: 66-74, 2016

Heyes D.J., Hardman S.J., Mansell D. et al.: Mechanistic reappraisal of early stage photochemistry in the light-driven enzyme protochlorophyllide oxidoreductase. - PLoS ONE 7: e45642, 2012.

Hogewoning S.W., Trouwborst G., Maljaars H. et al:: Blue light dose-responses of leaf photosynthesis, morphology, and chemical composition of Cucumis sativus grown under different combinations of red and blue light. - J. Exp. Bot. 61: 3107-3117, 2010.

Inoue S., Kinoshita T., Matsumoto M. et al.: Blue light-induced autophosphorylation of phototropin is a primary step for signaling. - P. Natl. Acad. Sci. USA 105: 5626-5631, 2008.

Love M.I., Huber W., Anders S.: Moderated estimation of fold change and dispersion for RNA-seq data with DESeq2. Genome Biol. 15: 550, 2014.

Johkan M., Shoji K., Goto F. et al:: Blue light-emitting diode light irradiation of seedlings improves seedling quality and growth after transplanting in red leaf lettuce. - HortScience 45:1809-1814, 2010.

Kaiser E., Morales A., Harbinson J. et al.: Dynamic photosynthesis in different environmental conditions. - J. Exp. Bot. 66: 24152426, 2015.

Kanehisa M., Araki M., Goto S. et al.: KEGG for linking genomes to life and the environment. - Nucleic Acids Res. 36: D480-D484, 2008.

Kim D., Langmead B., Salzberg S.L.: HISAT: a fast spliced aligner with low memory requirements. - Nat. Methods 12: 357-360, 2015.

Kim S.J., Hahn E.J., Heo J.W. et al.: Effects of LEDs on net photosynthetic rate, growth and leaf stomata of chrysanthemum plantlets in vitro. - Sci. Hortic. 101: 143-151, 2004.

Latowski D., Kuczyńska P., Strzałka K.: Xanthophyll cycle-a mechanism protecting plants against oxidative stress. - Redox Rep. 16: 78-90, 2011.

Li H.M., Lu X.M.: Effects of light quality on flowering, dynamic variation in physiological characteristics of pakchoi during budding and flowering stages. - Acta Bot. Boreal.-Occident. 
Sin. 36: 0730-0737, 2016. [In Chinese]

Li H.M., Tang C.M., Xu Z.G.: The effects of different light qualities on rapeseed (Brassica napus L.) plantlet growth and morphogenesis in vitro. - Sci. Hortic.-Amsterdam 150: 117124, 2013.

Liu X.Y., Chang T.T., Guo S.R. et al.: Effect of diferent light quality of LED on growth and photosynthetic cheracter in cherry tomato seedling. - Acta Hortic. 907: 325-330, 2011.

Livaka K.J., Schmittgen T.D.: Analysis of relative gene expression data using real-time quantitative PCR and the 2(-Delta Delta C(T)) Method. - Methods 25: 402-408, 2001.

Love M.I., Huber W., Anders S.: Moderated estimation of fold change and dispersion for RNA-seq data with DESeq2. Genome Biol. 15: 550, 2014.

Mao X., Cai T., Olyarchuk J.G., Wei L.: Automated genome annotation and pathway identification using the KEGG Orthology (KO) as a controlled vocabulary. - Bioinformatics 21: 3787-3793, 2005.

McDonald M.S.: Photobiology of higher plants. - In: McDonald M.S. (ed.): Photosynthesis-Physiological and Environmental Factors. Pp. 62-68. W. John \& Sons Ltd. Publ., London 2003.

Nanya K., Ishigami Y., Hikosaka S. et al.: Effects of blue and red light on stem elongation and flowering of tomato seedlings. Acta Hortic. 956: 264-266, 2012.

Nelson N., Yocum C.F.: Structure and function of photosystems I and II. - Annu. Rev. Plant Biol. 57: 521-565, 2006.

Nhut D.T., Takamura T., Watanabe H. et al.: Responses of strawberry plantlets cultured in vitro under superbright red and blue light-emitting diodes (LEDs). - Plant Cell Tiss. Org. Cult. 73: 43-52, 2003.

Pertea, M., Kim, D., Pertea, G. et al.: Transcript-level expression analysis of rna-seq experiments with hisat, stringtie and ballgown. - Nat. Protoc. 11: 1650-667, 2016.

Pimputkar S., Speck J.S., DenBaars S.P. et al.: Prospects for LED lighting. - Nat. Photonics 3: 180-182, 2009.

Reinbothe C., El-Bakkouri M., Buhr F. et al:: Chlorophyll biosynthesis: spotlight on protochlorophyllide reduction. Trends Plant Sci. 15: 614-624, 2010.

Rowe J.D., Griffiths W.T.: Protochlorophyllide reductase in photosynthetic prokaryotes and its role in chlorophyll synthesis. - Biochem. J. 311: 417-424, 1995.

Sæbø A., Krekling T., Appelgren M.: Light quality affects hotosynthesis and leaf anatomy of birch plantlets in vitro. Plant Cell Tissue Organ Cult. 41: 177-185, 1995.

Stenbaek A., Jensen P.E.: Redox regulation of chlorophyll biosynthesis. - Phytochemistry 71: 853-859, 2010.

Sytina O.A., Heyes D.J., Hunter C.N. et al.: Conformational changes in an ultrafast light-driven enzyme determine catalytic activity. - Nature 456: 1001-1004, 2008.

Trapnell C., Williams B.A., Pertea G. et al.: Transcript assembly and quantification by RNA-Seq reveals unannotated transcripts and isoform switching during cell differentiation.Nat. Biotechnol. 28: 511-515,2010.

Vasilev S., Bruce D.: Optimization and evolution of light harvesting in photosynthesis: the role of antenna chlorophyll conserved between photosystem II and photosystem I. - Plant Cell 16: 3059-3068, 2004.

Wang H., Gu M., Cui J. et al.: Effects of light quality on $\mathrm{CO}_{2}$ assimilation, chlorophyll-fluorescence quenching, expression of Calvin cycle genes and carbohydrate accumulation in Cucumis sativus. - J. Photochem. Photobiol. B 96: 30-37, 2009.

Wang J., Lu W., Tong Y.X., Yang Q.C.: Leaf morphology, photosynthetic performance, chlorophyll fluorescence, stomatal development of lettuce (Lactuca sativa L.) exposed to different ratios of red light to blue light. - Front. Plant Sci. 7: 250, 2016.

Wang P., Grimm B.: Organization of chlorophyll biosynthesis and insertion of chlorophyll into the chlorophyll-binding proteins in chloroplasts. - Photosynth. Res. 126: 189-202, 2015.

Wang X.Y., Xu X.M., Cui J.: The importance of blue light for leaf area expansion, development of photosynthetic apparatus, and chloroplast ultrastructure of Cucumis sativus grown under weak light. - Photosynthetica 53: 213-222, 2015.

Wollaeger H.M., Runkle E.S.: Growth of impatiens, petunia, salvia, and tomato seedlings under blue, green, and red lightemitting diodes. - HortScience 49: 734-740, 2014.

Xu D., Li J., Gangappa S.N. et al.: Convergence of light and ABA signaling on the ABI5 promoter. - PLoS Genet. 10: e1004197, 2014.

Young M.D., Wakefield M.J., Smyth G.K. et al.: Gene ontology analysis for RNA-seq: accounting for selection bias. Genome Biol. 11: R14, 2010.

(C) The authors. This is an open access article distributed under the terms of the Creative Commons BY-NC-ND Licence. 